\title{
Unitary dilation and fixed point theorem
}

\author{
By Marek Ptak (Kraków)
}

\begin{abstract}
In this paper certain fixed point theorems for functions on groups $Z^{\Omega}$ and $R^{\Omega}$ are proved. using unitary dilations.
\end{abstract}

Let $H$ be a Hilbert space. $L(H)$ denotes the space of all linear bounded operators in $H$. If $H$ is a subspace of a Hilbert space $K$, then $P_{H}$ denotes the (orthogonal) projection of $K$ onto $H$. The set of all functions from a set $\Omega$ into set $A$ with finite support is denoted by $A^{?} . R, Z$ stand for the sets of real integer numbers, respectively.

Let $G$ be a group (we only consider groups $D^{\Omega} \cdot R^{\Omega 2}$ ) with unit $e$. A functions $T(\cdot): G \rightarrow L(H)$ is called positice definite iff $T\left(s^{-1}\right)=T(s)^{*}$ for all $s$ in $G$ and

$$
\sum_{s \in G ;} \sum_{l \in G}\left(T\left(t^{-1} s\right) h(s), h(t)\right) \geqslant 0 \quad \text { for all } h \in H^{G} .
$$

A function $U(\cdot): G \rightarrow L(K)$ is called a unitary representation of $G$ in $K$ if $U(e)=I_{K}, U(s \cdot t)=U(s) \cdot U(t)$ for all $s, t \in G$ and $U(s)$ is a unitary operator in $L(K)$ for all $s \in G$. Let $T(\cdot): G \rightarrow L(H)$ be a function; then a unitary representation $U(\cdot): G \rightarrow L(K)$ is called a unitary dilation of $T(\cdot)$ if $H \subset K$ and $T(s)=\left.P_{H} U(s)\right|_{H}$ for all $s \in G$.

Dash, in [1], has proved a fixed point theorem for a positive definite sequence of operators indexed by integer numbers. In the present paper we prove fixed point theorems for a positive definite function on groups $Z^{\Omega}$ and $\boldsymbol{R}^{\Omega}$, using unitary dilations.

Let $\Omega$ be a set; for $\omega \in \Omega$ we define a function $e_{\omega}$ in $Z^{S 2}$ (or in $R^{\Omega}$ ) by $\boldsymbol{e}_{\omega}(\alpha)=1$ if $\alpha=\omega$, and $e_{\omega}(\alpha)=0$, otherwise. Now, we generalize the theorem from [1] to multi-parameter discrete functions.

THEOREM 1. Let $T(\cdot): Z^{\Omega} \rightarrow L(H)$ be a positive definite function on $Z^{\Omega}$ (the group of all functions from a set $\Omega$ into $Z$ with finite support) with $T(0)=1$. If for some $f$ in $H$ and all $\omega \in \Omega$

$$
T\left(e_{6}\right) f=f,
$$


then

$$
T(n) f=f \quad \text { for all } n \in Z^{\Omega} .
$$

Proof. $T(\cdot)$ is a positive definite, thus [Theorem I.7.1,2] there is $U(\cdot): Z^{\Omega} \rightarrow L(K)$, a unitary dilation of $T(\cdot)$, so that $T(n) g=P_{H} U(n) g$ for all $g$ in $H$ and all $n$ in $Z^{\Omega}$.

Let $\omega \in \Omega$; then

$$
\|f\|=\left\|T\left(e_{\omega}\right) f\right\|=\left\|P_{H} U\left(e_{\omega}\right) f\right\| \leqslant\left\|U\left(e_{\omega}\right) f\right\|=\|f\|,
$$

thus $U\left(e_{(j)}\right), f \in H$. For all $h \in H$

$$
(h, f)=\left(h, T\left(e_{\omega}\right) f\right)=\left(h, P_{H} U\left(e_{\omega}\right) f\right)=\left(h, U\left(e_{\omega}\right) f\right) .
$$

Hence $U\left(e_{\omega}\right) f=f$ and also $U\left(-e_{\omega}\right) f=U\left(e_{\omega}\right)^{-1} f=f$. If $n \in Z^{\Omega}$ then, because $U(\cdot)$ is a representation, $U(n)$ is a multiplication of a finite number of operators of the forms $U\left(e_{\omega}\right)$ or $U\left(-e_{\omega}\right)$ for some $\omega$ in $\Omega$. Thus $U(n) f=f$ for all $n \in Z^{\Omega}$. Hence

$$
T(n) f=P_{H} U(n) f=P_{H} f=f \quad \text { for all } n \in Z^{\Omega} .
$$

Now, we present a fixed point theorem for a function on the group $\boldsymbol{R}^{\Phi}$, It can be observed that:

Remark. Let $d_{k} \neq 0$ be a sequence converging monotonically to 0 and let $r \in \boldsymbol{R}$. Then there is a sequence $n_{k}$ of elements of $Z$ such that $d_{k} n_{k} \rightarrow r$ whenever $k \rightarrow \infty$.

$n_{k}$ can be defined as an integer number such that $\left|d_{k} n_{k}\right| \leqslant|r|$ and $\mid d_{k} n_{k}$ $-r \mid$ has the smallest value.

Note that $\boldsymbol{R}^{\Omega}$ is a topological group with pointwise convergence topology and that each element of $\boldsymbol{R}^{\Omega}$ can be multiplied pointwise by any functions from $\Omega$ to $R$.

TheOREM 2. Let $T(\cdot): \boldsymbol{R}^{\Omega} \rightarrow L(H)$ be a continuous in the strong operator topology in $L(H)$ positive definite function on $\boldsymbol{R}^{\Omega}$ (the group of all functions from a set $\Omega$ into $\boldsymbol{R}$ with finite support) such that $T(0)=I$ and let there be for some $f$ in $H$ a sequence $s_{k}(\cdot): \Omega \rightarrow R$, converging pointwisely to 0 , of non-vanishing functions such that $T\left(s_{k} \cdot e_{\omega}\right) f=f$ for all $k$ and $\omega$ in $\Omega$. Then

$$
T(s) f=f \text { for all } s \text { in } \boldsymbol{R}^{\Omega} \text {. }
$$

Proof. By Theorem I.7.1 of [2] there is $U(\cdot): R^{\Omega} \rightarrow L(K)$ a unitary dilation of $T(\cdot)$, thus $T(s) g=P_{H} U(s) g$ for all $g$ in $H$ and all $s \in R^{\Omega}$.

Like in Theorem 1, it can be proved that

$$
U\left(s_{k} \cdot n\right) f=f \text { for all } k \text { and all } n \text { in } Z^{\Omega} \text {. }
$$

Let $s \in \boldsymbol{R}^{\Omega}$ and denote by supps the support of $s$. We can choose from the sequence $s_{k}(\cdot)$ a subsequence, also denoted by $s_{k}(\cdot)$, such that $s_{k}(\omega)$ con- 
verges monotonically to 0 for all $\omega \in \operatorname{supp} s$, because the support of $s$ is finite. If $\omega \in \operatorname{supp} s$, then, by the remark above, there is a sequence $n_{k}^{s(\omega)}$ of elements of $\boldsymbol{Z}$ such that $s_{k}(\omega) \cdot n_{k}^{\mathrm{s}(\omega)} \rightarrow s(\omega)$ whenever $k \rightarrow \infty$. We define a sequence of functions $n_{k}^{s} \in Z^{\Omega}$ as follows: $n_{k}^{s}(\omega)=n_{k}^{s(\omega)}$ if $\omega \in \operatorname{supps}$, and $n_{k}^{s}(\omega)=0$ otherwise. Then $s_{k} \cdot n_{k}^{s} \in \boldsymbol{R}^{\Omega}$ and $s_{k} n_{k}^{s} \rightarrow s$ pointwise in $\boldsymbol{R}^{\Omega}$.

The representation $U(\cdot)$ is continuous in the strong operator topology in $L(K)$, because $T(\cdot)$ is such (Theorem I.7,1 [2]), thus $U(s) f=f$. Hence

$$
T(s) f=P_{H} U(s) f=P_{H} f=f \text {. }
$$

\section{References}

[1] A. T. Dash, Positice definite sequences of operators and a fixed point theorem. Canad. Math. Bull. 15 (2) (1972). 295.

[2] B. Sz. Nagý, C. Foias, Analyse harmonique des opiraterirs de respace de Hilberr. Masson et $\mathrm{C}^{\text {ie }}$, Academiai Kiado, 1967.

AKADEMIA ROLNICZA W KRAKOWIE

KATEDRA ZASTOSOWAŃ MATEMATYKI, KRAKÓW 